Applied Physiology, Nutrition, and Metabolism

Canadian Science Publishing Physiologie appliquée, nutrition et métabolisme

\title{
Achieving Canadian Physical Activity Guidelines is Associated with Better Vascular Function Independent of Aerobic Fitness and Sedentary Time in Older Adults.
}

\begin{tabular}{|r|l|}
\hline Journal: & Applied Physiology, Nutrition, and Metabolism \\
\hline Manuscript ID & apnm-2018-0033.R1 \\
\hline Manuscript Type: & Article \\
\hline Date Submitted by the Author: & 09-Mar-2018 \\
\hline Complete List of Authors: & $\begin{array}{l}\text { O'Brien, Myles; Dalhousie University, Kinesiology } \\
\text { Robinson, Susan; Dalhousie University, Kinesiology } \\
\text { Frayne, Ryan; Dalhousie University, Kinesiology } \\
\text { Mekary, Said; Acadia University, School of Kinesiology } \\
\text { Fowles, Jonathon; Acadia University, } \\
\text { Kimmerly, Derek; Dalhousie University, Kinesiology }\end{array}$ \\
\hline $\begin{array}{r}\text { Keyword: } \\
\text { Is the invited manuscript for } \\
\text { consideration in a Special } \\
\text { Issue? : }\end{array}$ & $\begin{array}{l}\text { Vascular ageing, Flow-mediated dilation, Sedentary behavior, physical } \\
\text { activity < exercise }\end{array}$ \\
\hline \hline
\end{tabular}




\section{Achieving Canadian Physical Activity Guidelines is Associated with Better Vascular} Function Independent of Aerobic Fitness and Sedentary Time in Older Adults.

Myles W. O’Brien ${ }^{1}$, Susan A. Robinson ${ }^{1}$, Ryan Frayne ${ }^{1}$, Said Mekary ${ }^{2}$, Jonathon R. Fowles ${ }^{2}$, Derek S. Kimmerly ${ }^{1}$

${ }^{1}$ Division of Kinesiology, School of Health and Human Performance, Faculty of Health,

Dalhousie University, Halifax, Nova Scotia, Canada

${ }^{2}$ School of Kinesiology, Acadia University, Wolfville, Nova Scotia, Canada

\section{Corresponding Author:}

Derek Stephen Kimmerly

School of Health and Human Performance, Division of Kinesiology

Dalhousie University, 6230 South Street, Halifax, Nova Scotia, Canada B3H 4R2

Phone: +1 (902) 494-2570

Email: dskimmerly@dal.ca

\section{Abstract Word Count: 242 \\ Manuscript Word Count (including References): 5246}

Tables: 3

Figures: 2 


\section{Abstract:}

Canadian physical activity guidelines recommend older adults accumulate 150 minutes of weekly moderate-vigorous physical activity (MVPA). Older adults who are insufficiently active may have reduced blood vessel health and an increased risk of cardiovascular disease. We tested this hypothesis in 11 older adults who did $(7 \bigcirc ; 65 \pm 5$ years; MVPA=239 \pm 81 minutes/week) and 10 older adults who did not $(7 q ; 68 \pm 9$ years; MVPA=95 \pm 33 minutes/week $)$ meet MVPA guidelines. Flow-mediated dilation (FMD) in the brachial (BA) and popliteal (POP) arteries, as well as, nitroglycerin-mediated dilation (NMD; endothelial-independent dilation) in the POP were assessed via ultrasonography. Aerobic fitness $\left(\mathrm{VO}_{2}\right.$ peak) was determined using a graded, maximal cycle ergometry test via indirect calorimetry. MVPA and sedentary time were assessed over five days using the PiezoRx ${ }^{\circledR}$ and activPAL ${ }^{\circledR}$, respectively. There were no differences in $\mathrm{VO}_{2}$ peak $\left(26 \pm 10\right.$ versus $22 \pm 10 \mathrm{mlO}_{2} / \mathrm{kg} / \mathrm{min}$; $\left.\mathrm{p}=0.26\right)$ or sedentary time $(512 \pm 64$ versus $517 \pm 76$ minutes/day; $p=0.87$ ) between groups; however, those who achieved the MVPA guidelines had a higher BA-FMD (5.1 $\pm 1.3 \%$ versus $3.6 \pm 1.7 \%$; $\mathrm{p}=0.03)$, POP-FMD $(2.6 \pm 1.1 \%$ versus $1.3 \pm 0.8 \%$; $\mathrm{p}=0.006)$ and POP-NMD $(5.1 \pm 1.7 \%$ versus $3.3 \pm 2.1 \% ; \mathrm{p}=0.04)$. In the pooled sample, MVPA was moderately-correlated to both BA-FMD ( $\mathrm{r}=0.53 ; \mathrm{p}=0.01)$ and POP-NMD $(\mathrm{r}=0.59 ; \mathrm{p}=0.005)$, and strongly-correlated to POP-FMD ( $\mathrm{r}=0.85 ; \mathrm{p}<0.001)$. Collectively, our results provide supporting evidence that meeting MVPA guidelines is associated with better vascular function and may reduce the risk of developing cardiovascular disease in older adults. Furthermore, these data suggest that weekly MVPA time may have a greater impact on blood vessel function than aerobic fitness and weekly sedentary time.

Key Words: Vascular Aging, Physical Activity, Sedentary Behavior, Flow-Mediated Dilation 


\section{Introduction:}

Regularly performed exercise may attenuate, or even prevent, the age-associated decrease in vascular function, reducing the risk of cardiovascular disease in older adults (Seals et al. 2009). Despite such benefits, only $12 \%$ of Canadian older adults achieve the national physical activity guidelines that recommend 150 minutes of moderate-vigorous physical activity (MVPA) per week (Statistics Canada 2015). Moreover, elderly persons typically spend the majority of their day engaged in sedentary behaviors (e.g., sitting or lying down; Harvey et al. 2015), which represents an increased risk for the development of cardiovascular complications independent of time spent in MVPA (Katzmarzyk et al. 2009). Older adults who meet the MVPA guidelines have a $30 \%$ decreased relative risk of morbidity and mortality, with the dose-response relationship demonstrating that further risk-reductions in morbidity and mortality are achieved $(\sim 60 \%)$ through increases in aerobic fitness (Paterson and Warburton 2010$)$.

Aging is associated with chronic low-grade inflammation and increased oxidative stress, which contributes to endothelial dysfunction and a decrease in nitric oxide (NO) bioavailability (Ungvari et al. 2010). The flow-mediated dilation (FMD) technique is a commonly used surrogate measure of vascular health, has been primarily performed in the brachial artery (BA) and can help predict the risk of future cardiovascular events (Inaba et al. 2010). However, heterogeneity exists in the FMD response of upper- and lower-limb arteries (Thijssen et al. 2011b), and the lower limb vasculature [e.g., popliteal artery (POP)] is more susceptible to the development of atherosclerosis (Debasso et al. 2004). BA-FMD and POP-FMD responses are both impaired in older adults, which has been partially attributed to diminished vascular smooth muscle responsiveness to NO (Parker et al. 2006). 
While aerobic training can augment conduit artery function and induce favorable structural adaptations in older adults (Thijssen et al. 2010), the relationship between habitual physical activity and sedentary behaviours on vascular function in this population is unclear. It has been observed that older adults who self-report higher levels of physical activity have either greater (Pierce et al. 2011; Walker et al. 2014) or similar (Siasos et al. 2013) BA-FMD compared to those who report less physical activity. A contributing factor to these discrepant findings may be that subjective measures of physical activity are generally unreliable when compared to objectives measures (e.g., pedometers and accelerometers) (Prince et al. 2008). Step and accelerometer counts are positively related to BA-FMD in patients with peripheral artery disease (Payvandi et al. 2010), and a 12-week pedometer-based interventional study demonstrated that 140 minutes per week of moderate-intensity physical activity improved BA-FMD in older adults (Suboc et al. 2014). However, to date, no study has directly compared vascular health in older adults who are, or are not, achieving the recommended MVPA guidelines. Furthermore, no study has adapted an all-encompassing approach, incorporating concurrent objective measures of aerobic fitness, physical activity and inclinometer determined sedentary activity.

One limitation of past research is that confounding factors known to influence vascular function, such as aerobic fitness and sedentary time, have not been concurrently assessed and/or fully considered (Franzoni et al. 2005; Suboc et al. 2016; Bailey et al. 2017). Most studies have focused on the upper-limb vasculature even though arteries in the lower-limb are more susceptible to the progression of atherosclerosis. As such, investigation into the relationship between aerobic fitness, habitual physical activity and sedentary times on lower-limb vascular function is warranted. 
The objective of this study was to compare upper- and lower-limb vascular function in older adults who, when objectively measured, achieve current MVPA guidelines versus those who do not. In addition, we investigated the correlational relationship between aerobic fitness, MVPA and sedentary time on endothelium-dependent (BA and POP) and -independent (POP only) vasodilation in older adults. We hypothesized that older adults who achieve the MVPA guidelines would demonstrate superior vasodilatory responses in both limbs. Furthermore, considering the direct relationship between changes in daily step counts and POP shear stress patterns (Boyle et al. 2013), we hypothesized that weekly MVPA and sedentary times will be related to lower-limb vascular function in older adults.

\section{Methods:}

\section{Participants:}

All protocols and procedures were approved by the Dalhousie University Health Sciences Research Ethics Board and the Acadia University Research Ethics Board. Twenty-one older ( $>55$ years), normotensive adults provided written, informed consent to participate in this study. Participants were dichotomized into those who achieved 150 minutes of objectively measured MVPA per week (Active, $n=11 ; 7+$ ) versus those who did not (Inactive, $n=10 ; 7 \circ$ ). Descriptive and activity-related data are presented in Table 1. Participants were cleared for MVPA using the Physical Activity Readiness Questionnaire Plus (Warburton et al. 2011). Three participants were on low-doses of blood pressure medication (Inactive: $n=2$; Active $n=1$ ) and one person in the Active group was asthmatic.

\section{Experimental Design:}

Participants reported for two separate laboratory sessions. Day 1 was dedicated to the assessments of BA and POP vascular function. On Day 2 they completed a graded, maximal 
cycling exercise test to determine aerobic fitness. Day 1 was conducted either prior to, or a minimum of 48 hours following the graded exercise test (see below for details). To minimize confounding influences on endothelial-dependent dilation, vascular assessments were performed 6 hours post-prandial, and participants avoided strenuous physical activity, as well as, the consumption of products known to acutely influence FMD responses (e.g., caffeine, chocolate, kiwi, saturated fats, folic acid supplements, antioxidant and multivitamin supplements) for 24 hours, consistent with FMD guidelines (Thijssen et al. 2011a). At least 48 hours following the determination of aerobic fitness and vascular health, physical activity and sedentary behaviors were measured over a 5-day period (see below for details). All study visits were performed in a thermoneutral environment $\left(21^{\circ} \mathrm{C}\right)$.

\section{Experimental Protocol:}

Anthropometrics \& Aerobic Fitness:

Height and body mass were measured using a calibrated stadiometer (Health-O-Meter, McCook Il, USA) to the nearest $0.5 \mathrm{~cm}$ and $0.1 \mathrm{~kg}$, respectively. Waist circumference was measured from the uppermost lateral border of the iliac crest and was recorded to the nearest 0.5 $\mathrm{cm}$. An incremental and maximal exercise test on a cycle ergometer (Lode Excalibur Sport, Groningen, The Netherlands) was administered to determine peak oxygen uptake $\left(\mathrm{VO}_{2} \mathrm{peak}\right)$ via a mixing chamber-based commercial metabolic system (TrueOne $2400^{\circledR}$, Parvomedics Inc., Sandy, UT). Following a 5-minute warm-up period of light-intensity cycling (30-50W), the workload was initially set at 1 watt per kilogram of body weight and gradually increased by 15 watts every minute until voluntary exhaustion. $\mathrm{VO}_{2}$ peak was determined as the highest 30 second averaged $\mathrm{VO}_{2}$ obtained during the test. Strong verbal encouragement was given throughout the test. Upon completion of the test, the workload was immediately reduced to the 
warm-up level for a 5-minute cool-down period. $\mathrm{VO}_{2}$ data were averaged over 15 -second intervals for the duration of the protocol.

\section{Activity Monitors:}

Participants wore a PiezoRx ${ }^{\circledR}\left(\right.$ StepsCount $^{\circledR}, \mathrm{ON}$, Canada $)$ and an activPAL ${ }^{\circledR}(\mathrm{Pal}$ Technologies Ltd. ${ }^{\circledR}$, Glasgow, UK) concurrently for five full days consistent with recommendations for physical activity and sedentary behaviour monitoring in older adults (Hart et al. 2011). Daily step counts and MVPA were assessed using the PiezoRx ${ }^{\circledR}$, which is a medical grade physical activity monitor that uses step rate thresholds to determine time spent in MVPA. The PiezoRx ${ }^{\circledR}$ is a valid measure of step count in older adults (Webber et al. 2014). Observations from our laboratory indicate the PiezoRx ${ }^{\circledR}$ is as accurate as tri-axial accelerometry for the determination of absolute MVPA (i.e., 3 METs and 6 METs) when adjusted to MVPA thresholds of 110 and 130 steps per minute, in adults and older adults (O'Brien et al. 2018). Placement of the PiezoRx ${ }^{\circledR}$ was standardized by securing it on their waistband or belt in line with their right mid-thigh as per manufactures recommendations. The activPAL ${ }^{\circledR}$ was waterproofed and secured using Tegaderm ${ }^{\mathrm{TM}}$ transparent medical dressing to the midline of their right thigh, one-third of the way between the hip and the knee. ActivPAL ${ }^{\circledR}$ protocols were based on previous research outlining important considerations for field-based research (Edwardson et al. 2016).

\section{Hemodynamics:}

Heart rate (HR) was determined via cardiac intervals obtained from lead II of a bipolar electrocardiography configuration. Brachial measurements of systolic blood pressure and diastolic blood pressure were recorded by an automated patient vital signs monitor (Carescape v100®, General Electric Healthcare). HR was sampled continuously at $1000 \mathrm{~Hz}$ using a PowerLab (PL3508 PowerLab 8/53, ADInstruments, Sydney, Australia) data acquisition system 
and displayed in real-time and analyzed offline using LabChart software (ADInstruments, Sydney, Australia).

\section{Vascular Measures:}

The right BA and left POP were imaged with participants in the supine and prone positions, respectively. The BA was imaged $3-5 \mathrm{~cm}$ proximal to the antecubital fossa and the POP was imaged proximal to the bifurcation at or slightly above the popliteal fossa. A pressure cuff attached to a rapid cuff inflation system (E20 and AG101, Hokanson ${ }^{\circledR}$, Bellevue, WA) was positioned $\sim 3 \mathrm{~cm}$ distal to the antecubital fossa (BA) or $\sim 10 \mathrm{~cm}$ distal to the popliteal fossa (POP). All images were obtained using a $12 \mathrm{MHz}$ multi-frequency linear array probe attached to a high-resolution ultrasound system (Vivid i, General Electric Healthcare). Simultaneous blood velocity signals were recorded in duplex mode at a pulsed frequency of $\sim 5 \mathrm{MHz}$ and corrected with an insonation angle of $60^{\circ}$ that remained constant throughout the study. The sample volume was adjusted for each participant such that the anterior to the posterior intima were included, as recommended in published guidelines (Thissen et al. 2011a).

Resting artery lumen diameter and blood flow velocity were measured for a minimum of two minutes before inflation of the pneumatic cuff. The pressure cuff was rapidly inflated to 250 $\mathrm{mmHg}$ for five minutes. Continuous arterial lumen diameter and blood flow velocity recordings were collected throughout the cuff inflation period. Upon release of cuff pressure, lumen diameter and velocity recordings continued for an additional five minutes. A minimum of 10 minutes separated the BA and POP FMD assessments to allow resting blood flow and shear rates to return to baseline levels. 
In addition to the FMD technique, the POP was imaged for 1-minute before and 10minutes following a sublingual administration of nitroglycerin $(0.4 \mathrm{mg})$ as an index of endothelial-independent vasodilation (Chen et al. 2002). At least 10 minutes of rest was provided between completion of the FMD assessments and the start of POP-NMD testing. Data Analysis:

Relative $\mathrm{VO}_{2}$ peak data were divided by $3.5 \mathrm{ml} / \mathrm{kg} / \mathrm{min}$ to calculate peak metabolic equivalents (Peak MET). A minimum 4 days of Valid PiezoRx ${ }^{\circledR}$ data (including 1 weekend day) were considered sufficient for analysis if a minimum 10 hours of wear time per day was achieved. These data were then adjusted to estimate one-full week of activity. Participants selfreported how many hours per day they wore the PiezoRx ${ }^{\circledR}$ and their time spent awake to accommodate activPAL ${ }^{\circledR}$ analysis of waking sedentary time. ActivPAL ${ }^{\circledR}$ data were exported from the activPAL ${ }^{\circledR}$ software (version 5.8.5) and analyzed using a customized LabVIEW program (LabVIEW 2013; National Instruments, Austin, TX) that summarized daily averages of awake time spent standing, sitting and lying down.

Vivid i ultrasound video signals were exported to an external laptop via a video graphics array converter (Epiphan Systems Inc., VGA 2 USB, Ottawa) for offline analysis. Analysis of artery diameter, blood flow velocity and shear rate (i.e., frictional forces of blood flow on the endothelium) were performed using automated commercial edge-detection and walltracking software (FMD Studio, Cardiovascular Suite, Quipu, Pisa, Italy). Vascular measurements were completed by MWO and SAR who demonstrated inter-tester coefficients of variation $(\mathrm{CV})$ of $1.1 \%, 4.3 \%$ and $3.9 \%$ for baseline diameter, $\mathrm{FMD} \%$ and $\mathrm{NMD} \%$, respectively. These CV values are consistent with previous research using automated edge-detection software (Ratcliffe et al. 2017). Blood flow was calculated as mean blood flow velocity $\times 60 \times \pi \times$ lumen 
radius $^{2}$. Relative FMD was calculated using the equation: FMD $(\%)=$ (post-cuff deflation peak diameter - baseline diameter)/baseline diameter $\times 100 \%)$. Shear rate $\left(\mathrm{SR}, \mathrm{s}^{-1}\right)$ was defined as $[4$ $\times$ Mean blood velocity $(\mathrm{cm} / \mathrm{s})] /$ diameter $(\mathrm{cm})$. Subsequently, the SR area under the curve $\left(\mathrm{SR}_{\mathrm{AUC}}\right)$ was calculated between the start of cuff deflation to the time that peak dilation occurred. To minimize the individual vasodilatory response to reactive hyperemia, $\mathrm{SR}_{\mathrm{AUC}}$ normalized FMD has been recommended (Padilla et al. 2008). However, neither BA-FMD nor POP-FMD were correlated to their respective $\mathrm{SR}_{\mathrm{AUC}}$ response (BA-FMD: $\mathrm{r}=0.29, \mathrm{p}=0.21$; POP-FMD: $r=-0.25, p=0.27$ ), suggesting the FMD-SR AUC $_{\text {to }}$ to discouraged (Atkinson et al. 2009). NMD was calculated as a percentage change from baseline to the peak lumen diameter obtained during the ten-minute period following sublingual administration of nitroglycerin.

\section{Statistical Analysis:}

Baseline characteristics, resting hemodynamics, pedometer wear time, $\mathrm{VO}_{2}$ peak, MVPA time, sedentary time, standing time and vascular measurements were compared between Active and Inactive groups using independent samples t-tests. Within group differences between BAFMD, POP-FMD and POP-NTG were calculated using a one-way ANOVA for both the Active and Inactive groups. The variance of differences was assessed using Mauchly's test of sphericity. Bonferroni post-hoc testing was conducted on statistically significant ANOVAs. Pearson's correlations were used to evaluate the relationship between $\mathrm{VO}_{2}$ peak, MVPA time and sedentary time with measures of vascular function in the pooled sample. Pearson correlations were interpreted as follows: $<0.5$ (weak), 0.5-0.7 (moderate) and $>0.7$ (strong) (Hinkle et al. 2003). All data were assessed for normality using a Shapiro-Wilk test and found to be normally distributed (all, $\mathrm{p}>0.1$ ). All statistics were completed in SPSS Version 23.0 (IBM, NY) statistical 
program. Statistical significance was accepted as $\mathrm{p}<0.05$. All data are presented as means \pm standard deviations (SD).

\section{Results:}

There were no differences (all $\mathrm{p}>0.05$ ) in age, waist circumference, body mass index, resting hemodynamics, $\mathrm{VO}_{2}$ peak or sedentary time between the Active and Inactive groups (Table 1). As shown in Table 1, those who met the MVPA guidelines accumulated 44\% more steps per day and participated in $87 \%$ more weekly MVPA (both, $p<0.001$ ) than those who did not. The Active and Inactive groups reported similar pedometer wear times of $13.7 \pm 1.3$ and $13.4 \pm 1.6$ hours/day $(\mathrm{p}=0.67)$, respectively.

\section{TABLE 1}

No group differences were noted for resting diameter, blood flow velocity or blood flow in either the BA or POP (all, $\mathrm{p}>0.05$; Table 2). The Inactive older adults had lower relative BAFMD $(3.58 \pm 1.72 \%$ vs. $5.09 \pm 1.27 \%$; $=0.03)$ and a lower POP-FMD $(1.27 \pm 0.81 \%$ vs. $2.62 \pm 1.14 \% ; \mathrm{p}=0.006)$ than those who achieved the minimum MVPA guidelines (Figure 1). Additionally, the Inactive group had lower POP endothelial-independent dilation response (3.34 $\pm 2.06 \%$ vs. $5.12 \pm 1.74 \% ; \mathrm{p}=0.04)$. As expected, POP-FMD was less than POP-NMD within groups (Active: $\mathrm{p}<0.001$, Inactive: $\mathrm{p}=0.01$ ) and POP-FMD was less than BA-FMD (both $\mathrm{p}<0.001)$

\section{TABLE 2}

\section{FIGURE 1}

The correlations between $\mathrm{VO}_{2}$ peak, MVPA, step count, standing time and sedentary time versus the vascular measures for the pooled sample are presented in Table $3 . \mathrm{VO}_{2}$ peak, MVPA and step count were all moderately correlated $(\mathrm{r}=0.53-0.63$; all $\mathrm{p}<0.05)$ to BA-FMD. Strong 
correlations $(r>0.70)$ were observed between POP-FMD and both MVPA $(r=0.85, p<0.001$; see Figure 2$)$ and daily step count $(\mathrm{r}=0.78, \mathrm{p}<0.001)$, while POP-FMD and $\mathrm{VO}_{2}$ peak were moderately correlated $(\mathrm{r}=0.59, \mathrm{p}<0.001)$. However, aerobic fitness was not correlated to NMD, but MVPA and step count were (MVPA: $r=0.59, \mathrm{p}=0.005$; step count: $\mathrm{r}=0.64, \mathrm{p}=0.002$ ). Sedentary time and standing time were not correlated to BA-FMD, POP-FMD or POP-NMD (all, $\mathrm{p}>0.05)$.

\section{TABLE 3}

\section{FIGURE 2}

\section{Discussion}

The purpose of this study was to compare upper- and lower-limb vascular health in older adults who achieved the Canadian physical activity guidelines of 150 minutes of weekly MVPA to age- and sex-matched peers who did not. The findings of this study suggest that older adults who met the MVPA guidelines have augmented endothelial-dependent (BA and POP) and endothelial-independent (POP) vasodilatory responses. Importantly, these superior vascular responses were not due to differences in aerobic fitness or weekly sedentary time. We also observed moderate-strong positive relationships between measures of physical activity (e.g., MVPA and daily step counts) with BA-FMD, POP-FMD and POP-NMD in the pooled sample of older adults. This represents the first evidence to verify that meeting the national guidelines for MVPA has a direct and beneficial effect on blood vessel health in an older population.

Although this is the first study investigating habitual activity on BA-FMD to incorporate an objective measure of physical activity and an inclinometer for sedentary activity, previous self-report studies are consistent with our findings. Walker and colleagues (2014) demonstrated that older adults who self-report being habitually active ( $>180$ minutes per week of vigorous 
exercise) had a higher BA-FMD response $(7.2 \pm 0.7 \%$ vs. $4.0 \pm 0.7 \%)$ than those who report minimal amounts of physical activity ( $<60$ minutes per week of exercise). Additionally, a 12week randomized control trial observed that achieving $\geq 20$ minutes per day of moderate physical activity, in bouts of $\geq 10$ minutes, was associated with a greater BA-FMD response in physically inactive older adults (Suboc et al. 2014). On the contrary, achieving 10000 steps per day, or $\geq 30$ minutes per days (not in bouts) did not result in BA-FMD improvements in their older population (Suboc et al. 2014). In keeping with these previous reports, our findings suggest that improvements in upper-limb endothelial function are largely influenced by the accumulation of more higher-intensity daily physical activity.

Our population is representative of a typical older Canadian with an average $\mathrm{VO}_{2}$ peak of 7 METs ("fair" to "good" range; CSEP 2013) (Statistics Canada, 2011) and 8.5 hours per day of sedentary time (Statistics Canada 2015). As such, older adults with poor aerobic fitness or those engage in excessive sedentary time (e.g., $>12$ hours/day) may demonstrate larger impairments in vascular function, independent of MVPA time. Certainly, increased MVPA is associated with greater aerobic fitness, perhaps, a larger sample of older adults who meet, and do not meet MVPA guidelines would observe differences in $\mathrm{VO}_{2}$ peak. This is especially of interest given the moderate correlations between $\mathrm{VO}_{2}$ peak to both BA-FMD and POP-FMD. However, our Active and Inactive groups had similar levels of aerobic fitness and sedentary time, which allowed us to isolate the independent effects of MVPA on vascular health.

This study is the first to investigate BA-FMD or POP-FMD responses in combination with objective physical and sedentary activity monitoring, which included a medical grade physical activity monitor and an inclinometer that distinguishes time spent standing (antisedentary behavior) from sitting and lying down. As such, future studies in this area are 
recommended to incorporate objective measures, because of the known social desirability bias of questionnaires assessing time spent engaging in physical activity and sedentary behaviors (Prince et al. 2008; Fowles et al. 2017). Discrepancies in subjective measures may, in part, explain the lack of differences observed by Siasos et al. (2013) in the FMD response of older adults classified as engaging in low $(4.8 \pm 2.6 \%)$, medium $(5.3 \pm 2.9 \%)$ or high $(5.9 \pm 4.1 \%)$ levels of physical activity. Interestingly, their BA-FMD responses $(4.8 \%$ to $5.9 \%)$ are similar to our Active group $(5.1 \pm 1.3 \%)$ and are marginally higher than the Inactive group $(3.6 \pm 1.7 \%)$. Furthermore, the POP-FMD data from our Inactive and Active groups are consistent with those observed by Nishiyama et al. (2008) (1.6 $\pm 0.5 \%)$ and Angerer et al. (2000) $(2.9 \pm 3.6 \%)$, respectively. Parker et al. (2006) observed a POP-NMD of $3.0 \pm 0.7 \%$ in a sample of older women. Despite their clinical relevance, no study to date has examined POP-FMD and POPNMD in male and female older adults.

One proposed mechanism behind the beneficial effects of meeting the MVPA guidelines are physical activity-induced increases in blood flow and shear stress, particularly in the lowerlimb vasculature (Pitsavos et al. 2005). Shear stress is the stimulus responsible for the production of NO and plays a primary role in the reduction of POP endothelial function observed during short-term reductions in daily physical activity levels (Boyle et al. 2013; Teixeira al. 2017). However, these previous studies did not investigate whether reductions in physical activity were associated with an increase in time spent sitting or lying down. In the present study, no differences were observed in the $\mathrm{SR}_{\mathrm{AUC}}$ response (i.e., an indication of microvascular function) despite a lower FMD response, indicative of reduced macrovascular function, in both arteries of the Inactive group. This may be attributed to age-associated increases in oxidative stress or endothelin-1 levels, which inhibit and counteract NO-induced vasodilation (Beckman et al. 1990; 
Donato et al. 2009). The age-associated increase in oxidative stress and endothelin-1 can be attenuated with regular aerobic exercise (Nyberg et al. 2013; Sallam and Laher 2016). It is plausible that achieving the minimum MVPA guidelines provided a sufficient stimulus to reduce oxidative stress and/or endothelin-1 concentration in the Active group (Ungvari et al. 2010), which may have contributed to their higher BA- and POP-FMD response. Additionally, oxidative stress reduces the bioconversion of nitroglycerin to NO within vascular smooth muscle cells (Mülsch et al. 2001; Münzel et al. 1995), which may partially explain the lower POP-NMD response of the Inactive group. More recently, higher levels of self-reported physical activity and measured aerobic fitness were associated with decreased endothelial cell senescence in older adults, which is proposed to be a contributing mechanism related to how exercise mitigates the age-associated decline in endothelial function (Rossman et al. 2017). However, the influence of physical activity and/or exercise on endothelial cell senescence has yet to be fully uncovered. Interestingly, BA and POP resting diameter, blood flow and shear rate were similar between the Active and Inactive groups. While some models of physical inactivity (e.g., 5 days of reduced step count and bed rest) observe decreases in arterial diameter (Bleeker et al. 2005; Boyle et al. 2013), vascular tone is regulated by many factors, which limits its generalizability as a measure of vascular structure (Thijssen et al. 2010). We did observe differences in nitroglycerin-induced maximal dilatory capacity in the popliteal artery, suggesting potential lower limb structural differences between the Active and Inactive groups. Specifically, the increased maximal dilatory capacity may be indicative of augmented vascular compliance in the presence of exogenous NO, potentially due to the positive effects of physical activity on elastin and collagen in older adults (Gates and Seals 2006). Thus, interventional studies should 
investigate the role of reduced physical activity, but preserved ST and aerobic fitness on localized and systemic conduit artery remodelling.

Although reductions in endothelial-dependent dilation were observed in the BA and POP, only POP endothelial-independent dilation was assessed. Therefore, it is unknown if upstream impairments in vasodilation are related to vascular smooth muscle dysfunction in the BA. The results of this study may be limited by sample size $(n=21)$ but it represents the first study to compare endothelial function in multiple vascular beds with objective measures of physical activity and sedentary behavior. Future research assessing the relationship between habitual physical activity and sedentary behaviors on vascular health is warranted, especially in clinical populations characterized by endothelial dysfunction who may benefit the most from increases in daily physical activity. Additionally, future research should investigate the impact of weekly MVPA on other known factors that influence vascular health in older adults, such as oxidative stress, endothelin-1, sex and diet.

Independent of aerobic fitness and sedentary time, older adults who do not achieve the Canadian physical activity MVPA guidelines have lower BA-FMD and POP-FMD responses compared to their more active peers, which can be partly attributed to decreased vascular smooth muscle sensitivity to NO, as suggested by the lower POP-NMD in the Inactive group. As well, measures of physical activity were positively-related to upper- and lower-limb vascular function, suggesting the need for population-focused interventions aimed at increasing the proportion of older adults who achieve MVPA guidelines as a means of promoting healthy aging and reducing the risk of cardiovascular diseases. 


\section{Conflict of Interest:}

The authors have no conflicts of interest to report.

\section{Acknowledgements:}

Support provided by: Canadian Foundation for Innovation: Leader's Opportunity Fund (DSK), Faculty of Health Professions Research Development (DSK), NSHRF Development/Innovation (DSK and SM) grants, as well as, the Acadia University McCain Foundation Fund (SM). MWO was supported by a Heart \& Stroke BrightRed Scholarship, NS Graduate Scholarship and an NSHRF Scotia Scholars Award. 


\section{References:}

Angerer, P., Negut, C., Störk, S., and von Schacky, C. 2001. Endothelial function of the popliteal artery in patients with coronary artery disease. Atherosclerosis, 155(1): 187-193.

PMID:11223441.

Atkinson, G., Batterham, A.M., Black, M.A., Cable, N.T., Hopkins, N.D., Dawson, E.A., et al. 2009. Is the ratio of flow-mediated dilation and shear rate a statistically sound approach to normalization in cross-sectionals tudies on endothelial function? J. Appl. Physiol. 107(6): 18931899. DOI: 10.1152/japplphysiol.00779.2009. PMID:19833808.

Bailey, T.G., Perissiou, M., Windsor, M., Russell, F., Golledge, J., Green, D.J. et al. 2017. Cardiorespiratory fitness modulates the acute flow-mediated dilation response following highintensity but not moderate-intensity exercise in elderly men. J. Appl. Physiol. 122(5): 1238-1248. DOI:10.1152/japplphysiol.00935.2016. PMID:28209742.

Beckman, J.S., Beckman, T.W., Chen, J., Marshall, P.A., and Freeman, B.A. 1990. Apparent hydroxyl radical production by peroxynitrite: implications for endothelial injury from nitric oxide and superoxide. Proc. Natl. Acad. Sci. USA, 87(4): 1620-1624. PMID:2154753.

Bleeker, M.W., Kooijman, M., Rongen, G.A., Hopman, M.T., and Smits, P. 2005. Preserved contribution of nitric oxide to baseline vascular tone in deconditioned human skeletal muscle. J. Physiol. 565: 685-694. DOI:10.113/jphysiol.2005.085936. PMID:15802292.

Boyle, L.J., Credeur, D.P., Jenkins, N.T., Padilla, J., Leidy, H.J., Thyfault, J.P., et al. 2013. Impact of reduced daily physical activity on conduit artery flow-mediated dilation and 
endothelial microparticles. J. Appl. Physiol. 115(10): 1519-1525.

DOI:10.1152/japplphysiol.00837.2013. PMID:24072406.

Chen, Z., Zhang, J., and Stamler, J.S. 2002. Identification of the enzymatic mechanism of nitroglycerin bioactivation. Proc. Natl. Acad. Sci. USA, 99(12): 8306-8311.

DOI:10.1073/pnas.122225199. PMID:12048254.

CSEP. 2013. Canadian Society for Exercise Physiology-Physical Activity Training for Health (CSEP-PATH). CSEP, Ottawa, Ont., Canada.

Debasso, R., Åstrand, H., Bjarnegård, N., Rydén Ahlgren, Å., Sandgren, T., and Länne, T. 2004. The popliteal artery, an usual muscular artery with wall properties similar to aorta: implications for susceptibility to aneurysm formation? J. Vasc. Surg. 39(4): 836-842.

DOI:10.1016/j.jvs.2003.12.005. PMID:15071452.

Donato, A.J., Gano, L.B., Eskurza, I., Silver, A.E., Gates, P.E., Jablonski, K., et al. 2009. Vascular endothelial dysfunction with aging: endothelin-1 and endothelial nitric oxide synthase. Am. J. Physiol. Heart Circ. Physiol. 297(1): H425-H432. DOI:10.1152/ajpheart.00689.2008. PMID:19465546.

Edwardson, C.L., Winkler, E.A.H., Bodicoat, D.H., Yates, T., Davies, M.J., Dunstan, D.W., et al. 2016. Considerations when using the activPAL monitor in field-based research with adult populations. J. Sport Health Sci. 6: 162-178. DOI:10.1016/j.jshs.2016.02.002.

Fowles, J.R., O’Brien, M.W., Wojcik, W.R., d’Entremont, L. and Shields, C.A. 2017. A pilot study: validity and reliability of the CSEP-PATH PASB-Q and a new leisure time physical 
activity questionnaire to assess physical activity and sedentary behaviours. Appl. Physiol. Nutr. Metab. 42(6): 677-680. DOI:10.1139/apnm-2016-0412. PMID:28264170.

Franzoni, F., Ghiadoni, L., Galetta, F., Plantinga, Y., Lubrano, V., Huang, Y., et al. 2005. Physical activity, plasma antioxidant capacity, and endothelium-dependent vasodilation in young and older men. Am. J. Hypertens. 18(4): 510-516. DOI:10.1016/j.amjhyper.2004.11.006. PMID:15831361.

Gates, P.E., and Seals, D.R. 2006. Decline in large elastic artery compliance with age: a therapeutic target for habitual exercise. Br. J. Sports Med. 40(11): 897-899.

DOI:10.1136/bjsm.2004.016782. PMID:16954129.

Hart, T.L., Swartz, A.N., Cashin, S.E., and Strath, S.J. How many days of monitoring predict physical activity and sedentary behavior in older adults? Int. J. Behav. Nutr. Phys. Act. 8:62. DOI:10.1186/1479-5868-8-62. PMID:21679426.

Harvey, J.A., Chastin, S.F., and Skelton D.A. 2015. How sedentary are older people? A systematic review of the amount of sedentary behavior. J. Aging Phys. Act. 23(3): 471-487. DOI:10.1123/japa.2014-0164. PMID:25387160.

Hinkle, D.E., Wiersma, W., and Jurs, S.G. 2003. Applied Statistics for Behavioural Sciences $5^{\text {th }}$ Edition. Houghton Mifflin, Boston, MA.

Inaba, Y., Chen, J.A., and Bergmann, S.R. 2010. Prediction of future cardiovascular outcomes by flow-mediated vasodilation of brachial artery: a meta-analysis. Int. J. Cardiovasc. Imaging. 26(6): 631-640. DOI:10.1007/s10554-010-9616-1. PMID:20339920. 
Katzmarzyk, P.T., Church, T.S., Craig, C.L., and Bouchard, C. 2009. Sitting time and mortality from all causes, cardiovascular disease, and cancer. Med. Sci. Sports Exerc. 41(5): 998-1005. DOI:10.1249/MSS.0b013e3181930355. PMID:19346988.

Mülsch, A., Oelze, M., Klöss, S., Mollnau, H., Töpfer, A., Smolenski, A., et al. 2001. Effects of in vivo nitroglycerin treatment on activity and expression of the guanylyl cyclase and cGMPdependent protein kinase and their downstream target vasodilator-stimulated phosphoprotein in aorta. Circulation, 103(17): 2188-2194. PMID:11331261.

Münzel, T., Sayegh, H., Freeman, B.A., Tarpey, M.M., and Harrison, D.G. 1995. Evidence for enhanced vascular superoxide anion production in nitrate tolerance. A novel mechanism underlying tolerance and cross-tolerance. J. Clin. Invest. 95(1):187-194. DOI:10.1172/JCI1 17637. PMID:7814613.

Nishiyama, S.K., Wray, D.W., and Richardson, R.S. 2008. Aging affects vascular structure and function in a limb-specific manner. J. Appl. Physiol. 105(5): 1661-1670.

DOI:10.1152/japplphysiol.90612.2008. PMID:18719234.

Nyberg, M., Mortensen, S.P., and Hellsten, Y. 2013. Physical activity opposes the age-related increase in skeletal muscle and plasma endothelin-1 levels and normalizes plasma endothelin-1 levels in individuals with essential hypertension. Acta. Physiol. 207(3): 524-535.

DOI:10.1111/apha.12048. PMID: 23227981.

O’Brien, M.W., Wojcik, W.R., d'Entremont, L., and Fowles, J.R. 2018. Validation of the PiezoRx ${ }^{\circledR}$ step count and moderate to vigorous physical activity times in free living conditions in adults: a pilot study. Int. J. Exerc. Sci. 11(7): 541-551. 
Padilla, J., Johnson, B.D., Newcomer, S.C., Wilhite, D.P., Mickleborough, T.D., Fly, A.D., et al. 2008. Normalization of flow-mediated dilation to shear stress area under the curve eliminates the impact of variable hyperemic stimulus. Cardiovasc. Ultrasound, 6: 44. DOI:10.1186/1476-71206-44. PMID:18771594.

Parker, B.A., Ridout, S.J., and Proctor, D.N. 2006. Age and flow-mediated dilation: a comparison of dilatory responsiveness in the brachial and popliteal arteries. Am. J. Physiol. Heart Circ. Physiol. 291(6): H3043:H3049. DOI: 10.1152/ajpheart.00190.2006. PMID:16861699.

Paterson, D.H., and Warburton, D.E. 2010. Physical activity and functional limitations in older adults: a systematic review related to Canada's physical activity guidelines. Int. J. Behav. Nutr. Phys. Act. 7:38. DOI:10.1186/1479-5868-7-38. PMID:20459782.

Payvandi, L., Dyer, A., McPherson, D., Ades, P., Stein, J., Liu, K., et al. 2009. Physical activity during daily life and brachial artery flow-mediated dilation in peripheral arterial disease. Vasc. Med. 14(3): 193-201. DOI:10.1177/1358863X08101018. PMID:19651668.

Pierce, G.L., Eskurza, I., Walker, A.E., Fay, T.N., and Seals, D.R. 2011. Sex-specific effects of habitual aerobic exercise on brachial artery flow-mediated dilation in middle-aged and older adults. Clin. Sci. 120(1): 13-23. DOI:10.1042/CS20100174. PMID:20642454.

Pitsavos, C., Panagiotakos, D.B., Chrysohoou, C., Kavouras, S., and Stefanadis, C. 2005. The associations between physical activity, inflammation, and coagulation markers, in people with metabolic syndrome: the ATTICA study. Eur. J. Cardiovasc. Prev. Rehabil. 12(2): 151-158. PMID:15785301. 
Prince, S.A., Adamo, K.B., Hamel, M.E., Hardt, J., Connor Gorber, S.C., and Tremblay, M. 2008. A comparison of direct versus self-report measures for assessing physical activity in adults: a systematic review. Int. J. Behav. Nutr. Phys. Act. 5: 56. DOI:10.1186/1479-5868-5-56. PMID:18990237.

Ratcliffe, B., Pawlak, R., Morales, F., Harrison, C., and Gurovich, A.N. 2017. Internal validation of an automated system for brachial and femoral flow mediated dilation. Clin. Hypertens. 23: 17. DOI:10.1186/s40885-017-0073-1. PMID:28852570.

Rossman, M.J., Kaplon, R.E., Hill, S.D., McNamara, M.N., Santos-Parker, J.R., Pierce, G.L., et al. 2017. Endothelial cell senescence with aging in healthy humans: prevention by habitual exercise and relation to vascular endothelial function. Am. J. Physiol. Heart Circ. Physiol. 313(5): H890-H895. DOI:10.1152/ajpheart.00416.2017. PMID:28971843.

Sallam, N., and Laher, I. 2016. Exercise modulates oxidative stress and inflammation in aging and cardiovascular diseases. Oxid. Med. Cell Longev. 2016: 7239639.

DOI:10.1155/2016/7239639. PMID:26823952.

Seals, D.R., Walker, A.E., Pierce, G.L., and Lesniewski, L.A. 2009. Habitual exercise and vascular ageing. J. Physiol. 587(23): 5541-5549. DOI:10.1113/jphysiol.2009.178822. PMID:19723776.

Siasos, G., Chrysohoou, C., Tousoulis, D., Oikonomou, E., Panagiotakes, D., Zaromitidou, M., et al. 2013. The impact of physical activity on endothelial function in middle-aged and elderly subjects: the Ikaria study. Hellenic J. Cardiol. 54(2): 94-101. PMID:23557608. 
Statistics Canada. 2011. Aerobic fitness in Canada 2007 to 2009. Health Fact Sheet. Statistics Canada Catalogue no. 82-625-X. Available from: https://www.statcan.gc.ca/pub/82-625x/2010001/article/11092-eng.htm.

Statistics Canada. 2015. Directly measured physical activity of adults, 2012 and 2013. Health Fact Sheet. Statistics Canada Catalogue no. 82-625-X. Available from: https://www.statcan.gc.ca/pub/82-625-x/2015001/article/14135-eng.htm.

Suboc, T.B., Strath, S.J., Dharmashankar, K., Coulliard, A., Miller, N., Wang, J., et al. 2014. Relative importance of step count, intensity, and duration on physical activity's impact on vascular structure and function in previously sedentary older adults. J. Am. Heart Assoc. 3(1): e000702. DOI:10.1161/JAHA.113.000702. PMID:24572255.

Suboc, T.B., Knabel, D., Strath, S.J., Dharmashanker, K., Coulliard, A., Malik, M., et al. 2016. Associations of reducing sedentary time with vascular function and insulin sensitivity in older sedentary adults. Am. J. Hypertens. 29(1): 46-53. DOI:10.1093/ajh/hpv063. PMID:25987647.

Teixeira, A.L., Padilla, J., and Vianna, L.C. 2017. Impaired popliteal artery flow-mediated dilation caused by reduced daily physical activity is prevented by increased shear stress. J. Appl. Physiol. 123(1): 49-54. DOI:10.1152/japplphysiol.00001.2017. PMID:28450547.

Thijssen, D.H., Maiorana, A.J., O’Driscoll, G., Cable, N.T., Hopman, M.T., and Green, D.J. 2010. Impact of inactivity and exercise on the vasculature in humans. Eur. J. Appl. Physiol. 108(5): 845-875. DOI:10.1007/s00421-009-1260-x. PMID:19943061.

Thijssen, D.H., Black, M.A., Pyke, K.E., Padilla, J., Atkinson, G., Harris, R.A., et al. 2011a. Assessment of flow-mediated dilation in humans: a methodological and physiological guideline. 
Am. J. Physiol. Heart Circ. Physiol. 300(1): H2:H12. DOI:10.1152/ajpheart.00471.2010. PMID:20952670.

Thijssen, D.H., Rowley, N., Padilla, J., Simmons, G.H., Laughlin, M.H., Whyte, G., et al. 2011 b. Relationship between upper and lower limb conduit artery vasodilatory function in humans. J. Appl. Physiol. 111(1): 244-250. DOI:10.1152/japplphysiol.00290.2011. PMID:21512151.

Ungvari, Z., Kaley, G., de Cabo, R., Sonntag, W.E., and Csiszar, A. 2010. Mechanisms of vascular aging: new perspectives. J. Gerontol. A. Biol. Sci. Med. Sci. 65(10): 1028-1041. DOI:10.1093/gerona/glq113. PMID:20576649.

Walker, A.E., Kaplon, R.E., Pierce, G.L., Nowlan, M.J., and Seals, D.R. 2014. Prevention of age-related endothelial dysfunction by habitual aerobic exercise in healthy humans: possible role of nuclear factor-кB. Clin. Sci. 127(11): 645-654. DOI:10.1042/CS20140030. PMID:24947434.

Warburton, D.E., Bredin, S.S., Jamnik, V., and Gledhill, N. 2011. Validation of the PAR-Q+ and ePARmed-X+. Health Fitness J. Can. 4(2): 38-46.

Webber, S.C., Magill, S.M., Schafer, J.L., and Wilson, K.C. GT3X+ accelerometer, Yamax pedometer, and SC-Step-MX pedometer step count accuracy in community-dwelling older adults. J. Aging Phys. Act. 22(3): 334-341. DOI:10.1123/japa.2013-0002. PMID:23921227. 
Table 1. Participant descriptive characteristics and activity-related data in those who met the MVPA guidelines (Active) and those who did not (Inactive).

\begin{tabular}{|c|c|c|}
\hline & $\operatorname{Active}(n=11)$ & Inactive $(n=10)$ \\
\hline Age (years) & $65 \pm 5[59-71]$ & $68 \pm 9[56-83]$ \\
\hline Sex (Male, Female) & $4 \hat{\jmath}, 7+$ & $3 \lesssim, 7+$ \\
\hline Body Mass Index $\left(\mathrm{kg} / \mathrm{m}^{2}\right)$ & $25.7 \pm 3.7[21-33]$ & $27.0 \pm 4.9[19-37]$ \\
\hline Waist Circumference $(\mathrm{cm})$ & $86.7 \pm 9.1[76-103]$ & $87.2 \pm 10.2[72-110]$ \\
\hline Heart Rate (beats/minute) & $69.9 \pm 11.1[55-90]$ & $67.2 \pm 11.2[50-83]$ \\
\hline Systolic Blood Pressure (mmHg) & $117.4 \pm 9.0[102-132]$ & $122.7 \pm 12.6[110-139]$ \\
\hline Diastolic Blood Pressure (mmHg) & $69.0 \pm 11.8[55-85]$ & $68.9 \pm 7.6[60-81]$ \\
\hline Mean Arterial Pressure (mmHg) & $84.3 \pm 8.7[70-97]$ & $86.0 \pm 7.8[77-99]$ \\
\hline $\mathrm{VO}_{2}$ peak $\left(\mathrm{mlO}_{2} / \mathrm{kg} / \mathrm{min}\right)$ & $26.1 \pm 10.3[13-45]$ & $21.7 \pm 6.3[14-34]$ \\
\hline Peak MET & $7.5 \pm 3.0[4-13]$ & $6.2 \pm 1.8[4-10]$ \\
\hline Step Count (steps/day) & $10602 \pm 2920 *[5318-15205]$ & $6760 \pm 1796[4747-9727]$ \\
\hline MVPA (mins/week) & $239 \pm 81 *[151-424]$ & $95 \pm 33[51-137]$ \\
\hline Standing Time (mins/day) & $396 \pm 75[257-485]$ & $391 \pm 85[288-550]$ \\
\hline Sedentary Time (mins/day) & $512 \pm 64[405-648]$ & $517 \pm 76[379-632]$ \\
\hline
\end{tabular}

Data are presented as means \pm SD [range]. MET, metabolic equivalent; MVPA, moderate-vigorous physical activity; $\mathrm{VO}_{2}$ peak, peak oxygen uptake. ${ }^{*}, \mathrm{p}<0.001$ vs. Inactive. 
Table 2. Brachial artery and popliteal artery measures at rest and from FMD testing between Active and Inactive older adults.

\begin{tabular}{lcccc}
\hline & \multicolumn{2}{c}{ Brachial Artery } & \multicolumn{2}{c}{ Popliteal Artery } \\
\hline & Active & Inactive & Active & Inactive \\
\hline Resting & & & & \\
Diameter (mm) & $4.07 \pm 0.56$ & $3.97 \pm 0.88$ & $6.18 \pm 1.37$ & $6.60 \pm 1.84$ \\
Blood Flow Velocity (cm/s) & $13.4 \pm 5.2$ & $12.4 \pm 4.9$ & $3.6 \pm 2.1$ & $4.3 \pm 1.7$ \\
Blood Flow (ml/min) & $100.3 \pm 32.7$ & $94.6 \pm 47.3$ & $61.1 \pm 35.2$ & $85.4 \pm 37.9$ \\
Shear Rate (s ${ }^{-1}$ ) & $137.9 \pm 65.3$ & $129.8 \pm 59.9$ & $26.0 \pm 19.8$ & $28.8 \pm 14.1$ \\
Flow-Mediated Dilation & & & & \\
Absolute FMD (mm) & $0.22 \pm 0.08 *$ & $0.14 \pm 0.07$ & $0.17 \pm 0.11^{*}$ & $0.08 \pm 0.05$ \\
SR & & & & \\
Auc & $12495 \pm 4358$ & $13212 \pm 3874$ & $5011 \pm 1982$ & $6032 \pm 2891$ \\
Time to peak dilation (s) & $67 \pm 20$ & $58 \pm 19$ & $109 \pm 13$ & $105 \pm 30$ \\
\hline
\end{tabular}

Data are presented as means $\pm \mathrm{SD}$. FMD, flow-mediated dilation; $\mathrm{SR}_{\mathrm{AUC}}$, shear rate area under the curve to peak dilation. ${ }^{*}, \mathrm{p}<0.05$ vs Inactive within the same artery.

Table 3. Correlations between measures of aerobic fitness, physical activity, standing and sedentary behavior to upper- and lower-limb vascular function.

Brachial FMD Popliteal FMD Popliteal NMD

\begin{tabular}{lccc}
\hline $\mathrm{VO}_{2}$ peak $\left(\mathrm{mlO}_{2} / \mathrm{kg} / \mathrm{min}\right)$ & $0.63^{*}$ & $0.66^{*}$ & 0.28 \\
MVPA (mins/week) & $0.53^{*}$ & $0.85 \dagger$ & $0.59^{*}$ \\
Step Count (steps/day) & $0.57^{*}$ & $0.78 \dagger$ & $0.64^{*}$ \\
Standing Time (mins/day) & -0.18 & -0.16 & 0.17 \\
Sedentary Time (mins/day) & -0.19 & -0.15 & -0.18 \\
\hline
\end{tabular}

Data are presented as Pearson product-moment coefficients $(\mathrm{r}) . \mathrm{VO}_{2}$ peak, peak oxygen uptake; MVPA, moderate-vigorous physical activity; FMD, flow-mediated dilation; NMD, nitroglycerin-mediated dilation. ${ }^{*}, \mathrm{p}<0.05 ; \uparrow, \mathrm{p}<0.001$. 


\section{List of Figure Captions:}

Figure 1. Comparison of brachial artery FMD, popliteal artery FMD and popliteal NMD between older adults who met the MVPA guidelines (Active) and those who did not (Inactive). Data are presented as means \pm standard deviations. FMD, flow-mediated dilation; NMD, nitroglycerinmediated dilation; ${ }^{*}, \mathrm{p}<0.05$ versus Inactive of same measure.

Figure 2. Pooled sample correlations between MVPA with brachial artery FMD (A), popliteal artery FMD (B) and popliteal artery NMD (C). MVPA, moderate-vigorous physical activity; FMD, flow-mediated dilation; NMD, nitroglycerin-mediated dilation. The triangles and circle symbols represent Inactive and Active subjects, respectively. 


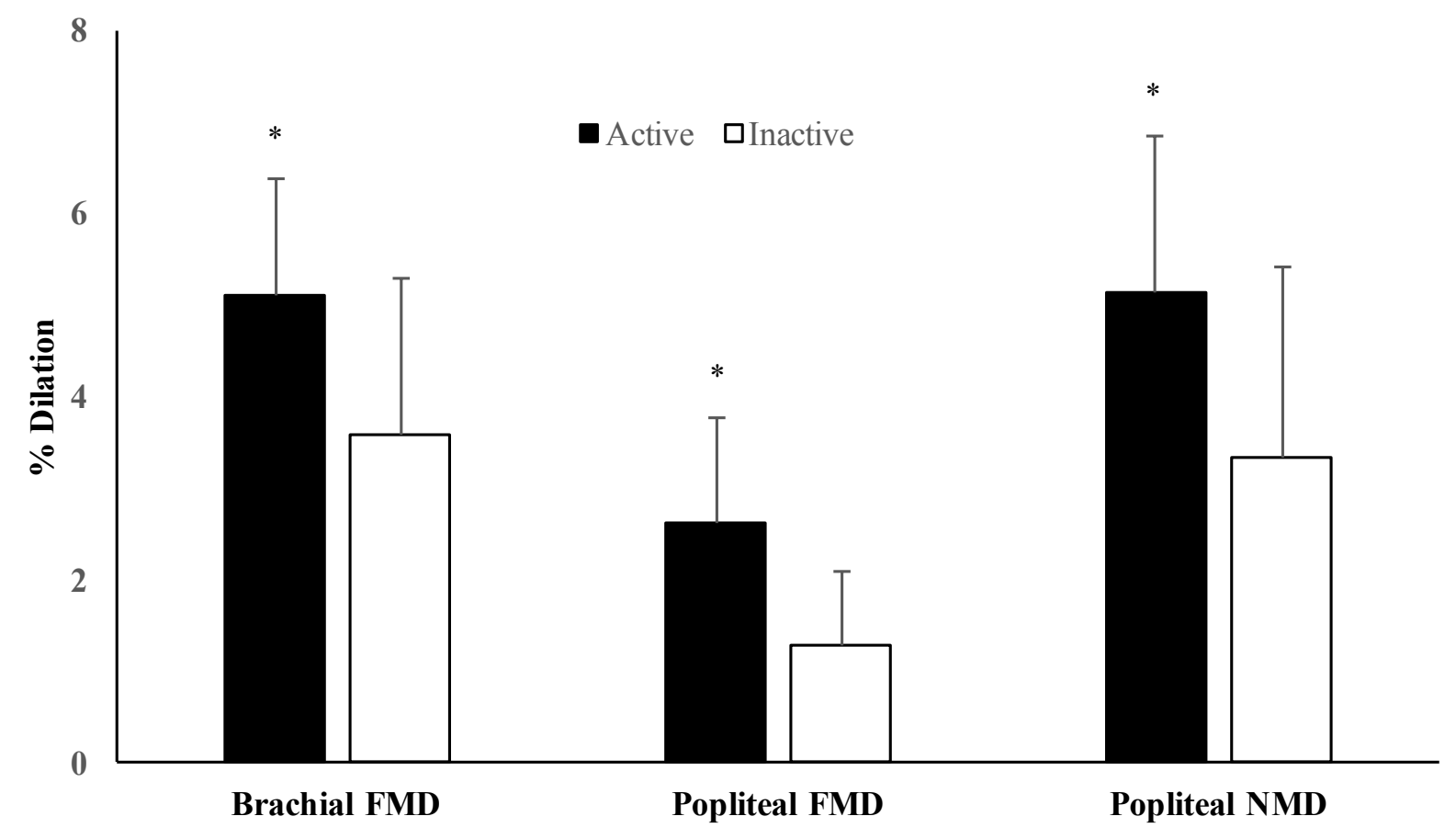



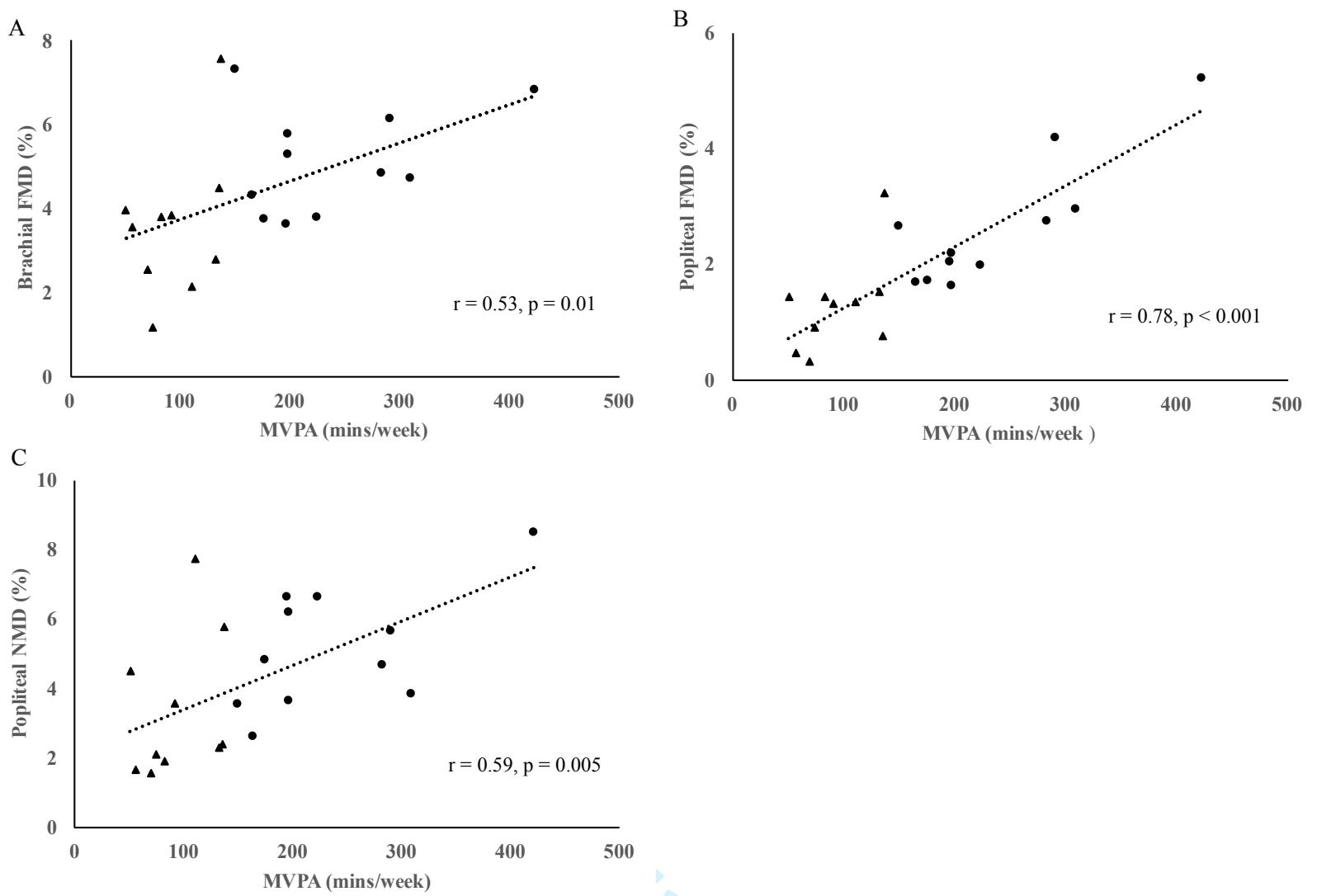